\title{
Pleuropulmonary Blastoma Mimicking Lobar Pneumonia with DICER1 Gene Positive in a 3-Year-Old Child: A Case Report and Review of the Literature
}

\section{Essa Alrashidi ${ }^{1}$, Ahmed Al Enizi ${ }^{2}$, Eatedal Ghareeb ${ }^{3}$, Abdelbasit Elsayed Ali ${ }^{4}$, Waleed Alharthi ${ }^{5}$, Mohammad Al Ayyaf ${ }^{6}$, Kris Ann P Schultz ${ }^{7}$ and Wael H Muharram ${ }^{8}$}

${ }^{1}$ Consultant, Pediatric Pulmonology, King Saud Medical City, Saudi Arabia

${ }^{2}$ Consultant, Pediatric Pulmonology and Sleep Medicine, King Saud Medical City, Saudi Arabia

${ }^{3}$ Consultant, Pediatric Hematology and Oncology, King Saud Medical City, Saudi

Arabia

${ }^{4}$ Consultant, Pediatric Surgery, King Saud Medical City, Saudi Arabia

${ }^{5}$ Assistant Consultant, Pediatric Pulmonary Department, King Saud Medical City,

Saudi Arabia

${ }^{6}$ Consultant, Histopathology, King Saud Medical City, Saudi Arabia

${ }^{7}$ International PPB/DICER1Registry and Cancer and Blood Disorder, Childrens

Minnesota, Minneapolis, MN, USA

${ }^{8}$ Consultant General Pediatric, King Saud Medical City, Saudi Arabia

*Corresponding Author: Abdelbasit Elsayed Ali, Consultant, Pediatric Surgery, King

Saud Medical City, Saudi Arabia.
Received: May 14, 2021

Published: June 15, 2021

(C) All rights are reserved by Abdelbasit

Elsayed Ali., et al.

\begin{abstract}
Introduction: Pleuropulmonary blastoma is a very rare malignant tumor of the lung presenting in early childhood of less than 6 years.

Case Presentation: A 3-years-old Saudi girl with a family history of malignancy presented with a history of a cough for one week and fever for 5 days. Radiological evaluation indicated a right lung mass. Thoracotomy and excisional biopsy showed a solid variant of pleuropulmonary blastoma. DICER1 gene was detected. Surgical treatment followed by chemotherapy resulted in disease clearance. Conclusion: To the best of our knowledge, this is the first reported pediatric case of DICER1 gene positive in our country. This case is reported to highlight the importance of attention to detailed history and early intervention in suspected cases. In the presence of persistent respiratory symptoms with heterogeneous lesions seen on chest imaging, the suspicion of malignant lung disease should be considered. Aggressive surgical resection and adjunctive chemotherapy can decrease progression in advanced disease.
\end{abstract}

Keywords: Pleuropulmonary Blastoma; Lung, Pneumonia; Pediatric; DICER1

\section{Abbreviations}

PPB: Pleuropulmonary Blastoma; CXR: Chest X-Ray; MRI: Magnetic Resonance Imaging; GIA Stapler: Gastrointestinal Anastomosis Stapler; MSA: Mammary Serum Antigen; CD99 Antigen: Cluster of Differentiation 99

\section{Introduction}

Although pulmonary malignancy of childhood is uncommon, Pleuropulmonary blastoma (PPB) is the most common primary lung malignancy seen and reported almost exclusively in early 
Pleuropulmonary Blastoma Mimicking Lobar Pneumonia with DICER1 Gene Positive in a 3-Year-Old Child: A Case Report and Review of the Literature

childhood with some adult reports in the literature [1]. PPB is a rare dysontogenetic pulmonary malignancy with highly aggressive behavior and some metastasis. There are four pathological types described based on the tissue morphology: type I or (cystic type), type II or (cystic/solid type) and type III or (solid type). The progression of type I to types II and III is well documented [2]. Lastly Type Ir (regressed) which is a unique, purely cystic type due to absences of primitive cell component [3]. This case is reported to increase awareness about this rare condition and emphasize the importance of taking detailed past and present history.

\section{Case Presentation}

We present a 3 years old Saudi girl who was previously healthy. She was admitted to a general pediatric ward as a case of complicated pneumonia with effusion. There was history of fever associated with chills and rigors which responded partially to antipyretic medication, with history of wet cough for 2 weeks. One month earlier, there was history of chronic right sided chest pain in the fifth intercostal space mid-axillary line with no improvement with analgesia. There was no history of weight loss or contact with a sick patient, however, there was a positive family history of renal and brain tumors. On clinical examination, she was ill and febrile with signs of respiratory distress, tachypnea, dyspnea with markedly reduced right sided chest movement with respiration, dullness on percussion and diminished air entry on ipsilateral auscultation. CXR showed a marked opacity in almost all of the right side of the chest with a leftwards mediastinal shift (Figure 1).

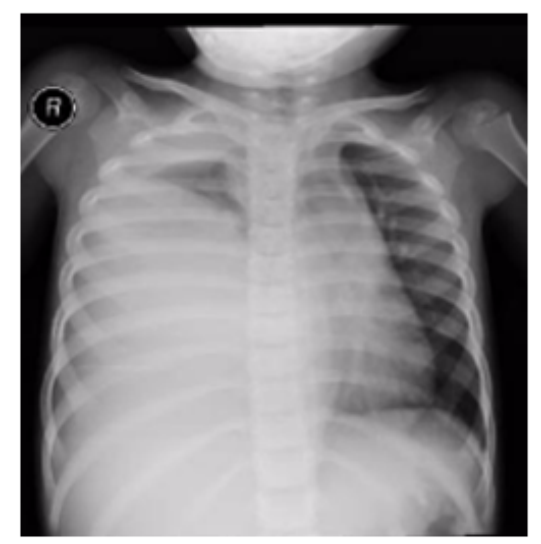

Figure 1: CXR showing Rt sided marked opacity of the middle and lower zones with mediastinal shift.

A chest ultrasound showed evidence of mild to moderate right pleural effusion with a heterogeneous lung mass lesion measuring
$6.7 \times 7.8 \mathrm{~cm}$ in the right lower chest, while abdominal us scan findings were within normal.

A chest CT scan showed a $10 \times 10 \times 7$ centimeter irregular mass lesion with mixed density, but predominantly hypo dense, seen in the region of the right middle lobe with associated pleural effusion and a marked left mediastinal shift (Figure 2a and 2b). Abnormal tumor vessels were seen within the upper portion of the mass. Residual pneumatized lung seen in the right upper lobe, while the other parts of the lung appeared compressed and collapsed. There was no gross mediastinal lymphadenopathy. The left lung was normal and with no pleural effusion. No pneumothorax or pericardial effusion were detected. No evidence of metastasis was found on renal ultrasound, brain MRI and bone scintigraphy.

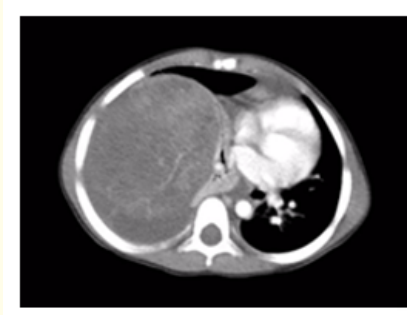

$2 \mathrm{a}$

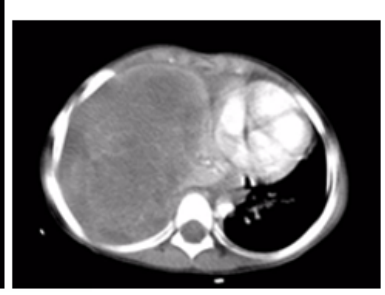

$2 \mathrm{~b}$
Figure 2: CT chest showed a $10 \times 10 \times 7$ centimeter, irregular mixed density mass lesion in the origin of the right middle lobe with associated pleural effusion (a), marked mediastinal shift (b) and right diaphragmatic depression. Abnormal tumoral vessels is seen within the upper portion of the mass. Residual pneumatized lung seen in the right upper and middle lobe, while the other parts of the lung appear compressed and collapsed. No gross mediastinal lymphadenopathy. Normal left lung with no pleural effusion. No pericardial effusion, no pneumothorax.

Surgical removal of the mass was done following appropriate preoperative preparation. At operation, under general anesthesia and aseptic environment, through the $6^{\text {th }}$ right intercostal space thoracotomy, thick pleura was encountered and a $200 \mathrm{ml}$ of altered hemorrhagic pleural effusion was drained. There was a large, friable fungating mass arising from and entirely replacing the right middle lobe (Figure 3), occupying almost two thirds of the right hemi-thoracic space, displacing and compressing what were apparently grossly healthy right upper and lower lobes. It was ad- 
herent to the pleura and a tumor nodule was attached to the right hemi-diaphragm. The mass was excised following control of the pedicle with a GIA stapler and 2-0 silk ligatures and the full thickness diaphragmatic nodule was removed with the defect repaired with 2-0 Vicryl. At the end of the procedure, complete macroscopic clearance of the tumor fragments was achieved (Figure 4-6). The mass weighed approximately $500 \mathrm{gm}$. Patient had a size $16 \mathrm{Fr}$ chest drain inserted through a separate stab incision. She had an uneventful postoperative recovery and the chest drain was removed 4 days later (Figure 7).

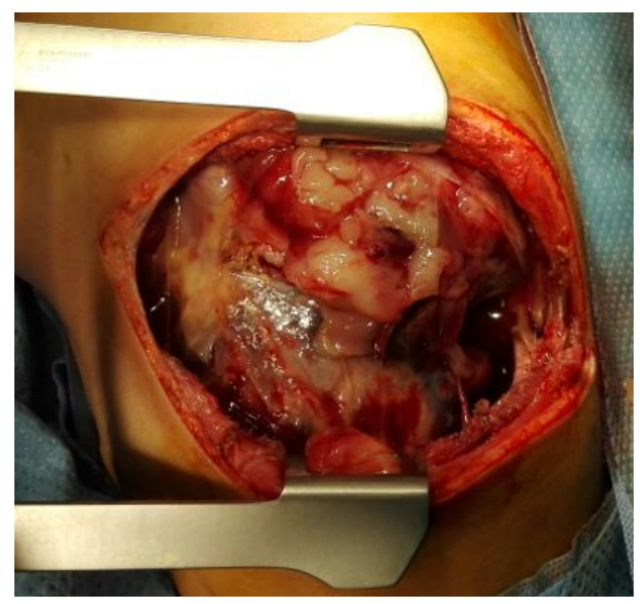

Figure 3: Intraoperative demonstration of a large fungating right lung mass.

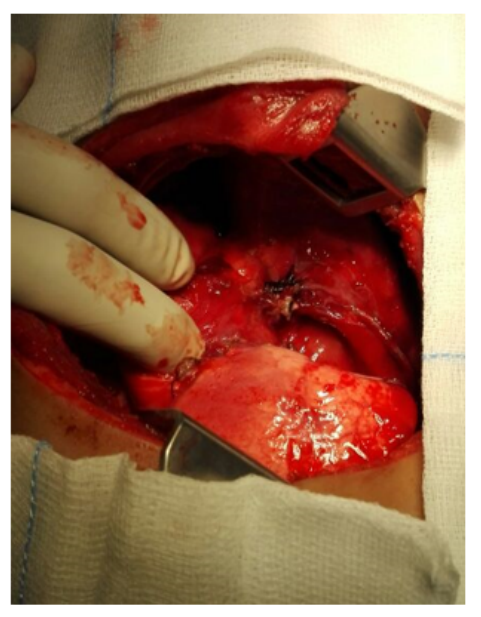

Figure 4: Following excision of the middle lobe mass, illustration shows resection margin on the remaining right upper and lower lung lobes.

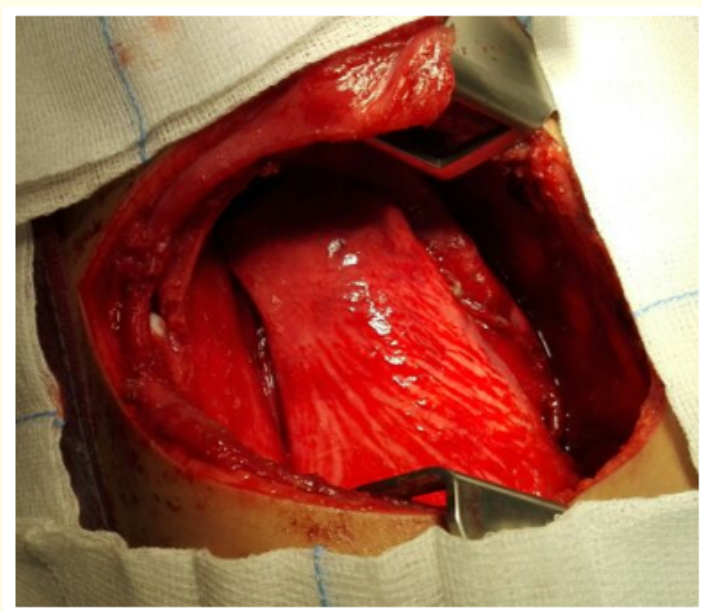

Figure 5: Right lower and upper lung lobes starting to inflate.

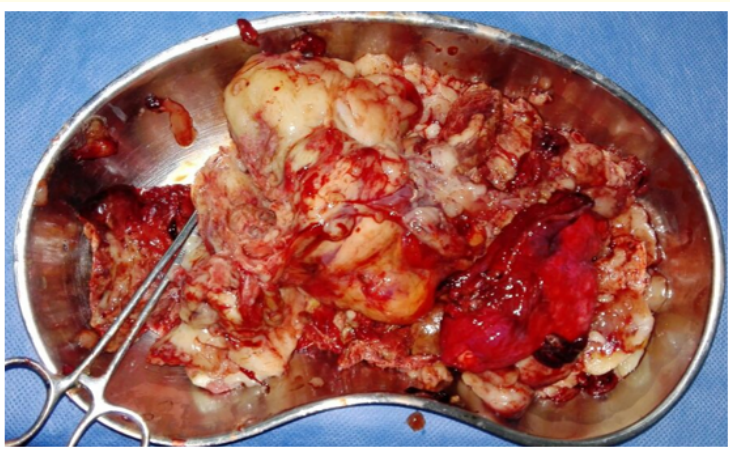

Figure 6: Shows the excisional biopsy of a fragmented right middle lobe tumor tissue.

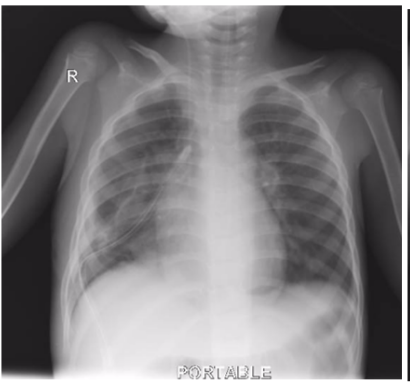

$7 \mathrm{a}$

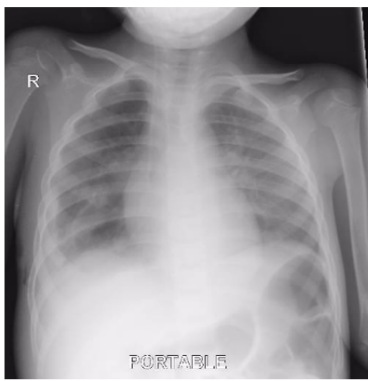

$7 \mathrm{~b}$
Figure 7a and 7b: Postoperative CXR showing disappearance of the right lung field opacity a. with the chest drain in place. b. following removal of the chest drain. 
Pleuropulmonary Blastoma Mimicking Lobar Pneumonia with DICER1 Gene Positive in a 3-Year-Old Child: A Case Report and Review of the Literature

The histopathology report showed a fragmented tumor tissue measuring together 11 X 10 X $6 \mathrm{~cm}$. Microscopic study revealed a malignant tumor composed of primitive blastema-like cells with hyperchromatic nuclei, high nucleocytoplasmic ratio and abundant mitoses. There were spindle cells embedded in myxoid stroma, a group of immature chondroid elements, single and groups of large anaplastic cells and area of hemorrhage and necrosis seen. No lymphovascular or perineural invasion seen. (Figure $8 \mathrm{a}$ and $8 \mathrm{~b}$ ). Fragments of pleural tissue with the tumor and a few fragments of emphysematous lung involved by the tumor were also seen.

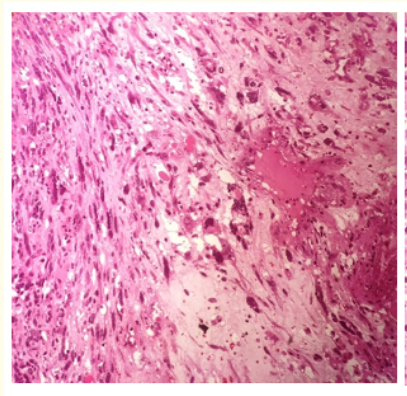

$8 \mathrm{a}$

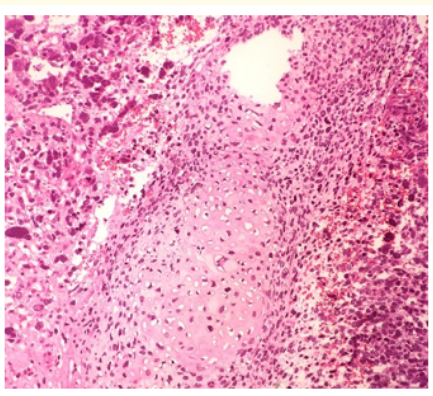

$8 \mathrm{~b}$
Figure 8a and 8b: Sections show a malignant tumor composed of premetive blastema like cells with hyperchromatic nuclei, high nucleocytoplasmic ratio and abundant mitosis. The spindle cells are embedded in myxoid stroma (a). Groups of immature chondroid elements (b).

Immunohistochemistry proved strong and diffuse positive vimentin, focal positivity for desmin and MSA and was weakly positive for CD99. Synaptophysin was negative.

Our case was reviewed by histopathology department of pleuropulmonary blastoma registry and confirmed the diagnosis of a type III (solid variant) right lung Pleuropulmonary blastoma (PPB T-III).

Screening for DICER1 gene mutation was done and proved to be positive. Subsequent test on all family members showed positive gene mutation in the mother and one of two siblings.

The patient started on chemotherapy as per pleuropulmonary blastoma registry guidelines (2016) for type III, and treatment continued for 36 weeks. During the course of treatment, complete res- olution of the previous right sided small amount of residual pleural effusion was noted which reflects excellent response to treatment.

Follow up bone scan (Figure 9), brain MRI (Figure 10), chest CT scan (Figure 11) and CXR (Figure 12) up to 3 years postoperatively were unremarkable.

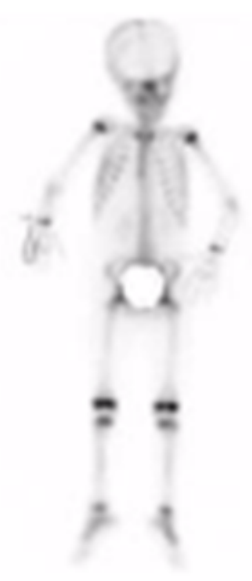

Figure 9: Follow up bone scan showing a negative study for bone metastases.
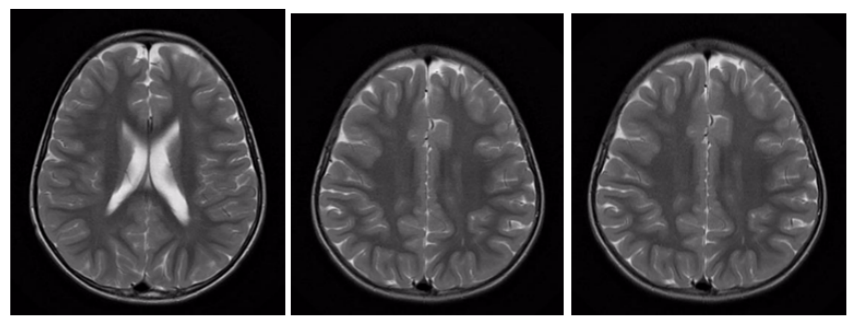

Figure 10: Follow up brain MR scan 6 months postoperatively. Unremarkable brain MRI, no abnormal enhancement or evidence of brain metastasis.

\section{Discussion}

Pleuropulmonary blastoma, also referred to as: cystic mesenchymal hamartoma, mesenchymal cystic hamartoma, pneumoblastoma, PPB, pulmonary rhabdomyosarcoma, rhabdomyosarcoma in lung cyst; or CPAM/CCAM type 4 [3], is a rare childhood malignant dysontogenetic intrathoracic neoplasm of pulmonary and/or pleu- 


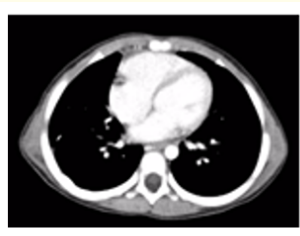

$11 \mathrm{a}$

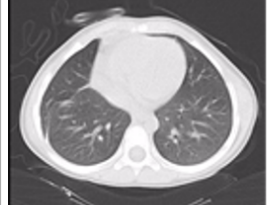

$11 b$

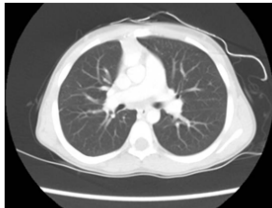

$11 \mathrm{c}$
Figure 11a-11c: Follow up chest CT scan 6 months (a and b), and 3 years (c) postoperatively. No definite residual or recurrent soft tissue mass at the site of previous pleuropulmonary blastoma could be appreciated in these exams.

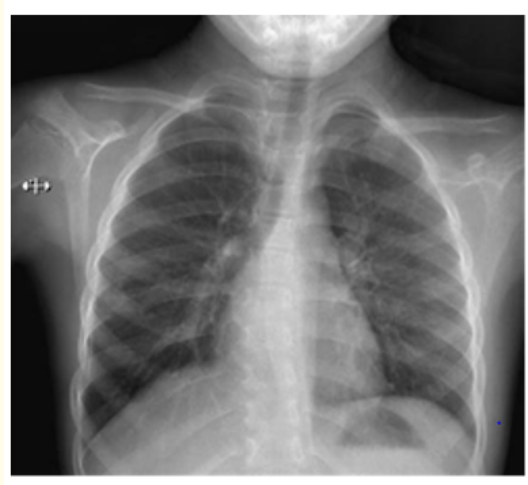

Figure 12: Three years post-surgical and chemotherapeutic treatment follow up CXR, showing unremarkable study. No definite residual or recurrent mass could be appreciated in this exam.

ral mesenchyme, representing around $15 \%$ of all primary pediatric pulmonary tumors [4]. Morphologically, PPB has four types; type 1 with cystic tumors, type II with solid and cystic types, and type III with solid tumors [5,6], type Ir (regressed) unique purely cystic type is due to absences of primitive cell component [7]. DICER1 Germline gene mutation has been considered a major etiologic factor [5,8-11]. The presence of a germline mutation in the DICER1 gene as lead for familial cases of PPB, was first noted in 2009 [12] and was followed by more studies to explain the role of DICER1 in PPB [13-15].
There are variations in clinical presentation and radiological features that may be predictive for PPB, including symptoms of pneumothorax and may manifest as tension pneumothorax [16] and bilateral or multi-segment involvement [17]. PPB may cause mediastinal shift and a mass effect on the adjacent structures [18].

When an opacity on a CXR is suspicious of a lung mass, ultrasound and CT scan of a suspected PPB may demonstrate a cystic or a purely solid lesion. Our case showed a heterogeneous mass lesion which is compatible with what is reported in the literature [19]. It is crucial to have an early histological recognition and differentiation from congenital lung and airway malformations and other benign cysts. Delay in the diagnosis and late onset treatment can lead to progress of the tumor and potential complications of advanced disease. In addition to local recurrence, PPB have metastatic disease, in central nervous system, bone, eye and rarely liver $[4,5,18-20]$.

To identify a lesion of PPB correctly, it may prove difficult for even experienced pathologists, so a central pathology review is crucial in clarifying questionable cases $[2,5,19]$.

There is insufficient data about pediatric PPB and the number of patients around the world is small so treatment guideline depends on the institution and treating physician. Generally speaking, the outcome and prognosis depend on the histological type of PBB; type Ir is better than the other types [5].

Suggested treatment options vary depending on the stage of PPB. Complete surgical resection and watchful waiting may be considered the best treatment options for (type I and type IR), however, in case of Type II and type III, complete surgical resection is the mainstay of treatment and decision of chemotherapy is determined by the physicians and the institution [21-24].

Pleuropulmonary blastoma has a poor prognosis, however, there are reports that the disease may be responsive to aggressive multimodal therapy [20-22]. Surgical resection and intensive chemotherapy are recommended for type II and type III PPB.

Curative role of radiotherapy for PPB is unclear and no case reports or studies specifically support that but may be used in case of residual PBB after surgical resection and chemotherapy [21,24,25].

\section{Conclusion}

This case is reported to highlight the importance of attention to detailed history and early intervention in suspected cases. In the 
Pleuropulmonary Blastoma Mimicking Lobar Pneumonia with DICER1 Gene Positive in a 3-Year-0ld Child: A Case Report and Review of the Literature

presence of persistent or intermittent respiratory symptoms with heterogeneous lesions seen on chest imaging, the suspicion of malignant lung disease, including PPB, should not be underestimated. Surgical resection and adjunctive chemotherapy decrease progression compared with surgery as a lone treatment.

\section{Declaration}

Written informed consent was obtained from the patient's parent for publication of this case report and the accompanying images. A copy of the written consent is available for review by the Editor-in-Chief of the journal if required.

\section{Funding Support}

No funding was received for this study.

\section{Competing Interests}

The authors declare that have no competing interests.

\section{Authors' Contributions}

All authors were involved in the writing and editing of the manuscript. All authors read and approved the final manuscript.

\section{Financial Disclosure}

The author have indicated they have no financial relationships relevant to this article to disclose.

\section{Bibliography}

1. Dehner LP. "Pleuropulmonary blastoma is THE pulmonary blastoma of childhood". Seminars in Diagnostic Pathology 1.2 (1994): 144-151.

2. Hill DA., et al. "Type I pleuropulmonary blastoma: pathology and biology study of 51 cases from the international pleuropulmonary blastoma registry Review". American Journal of Surgical Pathology 32.2 (2008): 282-295.

3. Mlika M., et al "Pleuropulmonary Blastoma". StatPearls [Internet] Treasure Island (FL): StatPearls Publishing (2021).

4. Khan AA., et al. "Pleuropulmonary Blastoma: A Case Report and Review of the Literature". Case Reports in Pathology (2014).

5. Messinger YH., et al. "Pleuropulmonary blastoma: a report on 350 central pathology-confirmed pleuropulmonary blastoma cases by the International Pleuropulmonary Blastoma Registry". Cancer 121.2 (2015): 276-285.
6. Zhang N., et al. "Childhood pleuropulmonary blastoma: A clinicopathologic study of 16 cases". Zhonghua Bing Li Xue Za Zhi 43 (2014): 747-752.

7. Messinger YH., et al. "Outcome of 116 cases of pleuropulmonary blastoma type I and type Ir (regressed): A report from the International PPB Registry (IPPBR)". Journal of Clinical Oncology 30S (2012): 9522.

8. Van Engelen K., et al. "DICER1 syndrome: Approach to testing and management at a large pediatric tertiary care center". Clinical Trial Pediatric Blood Cancer 65.1 (2018).

9. Cai S., et al. "DICER1 mutations in twelve Chinese patients with pleuropulmonary blastoma”. Science China Life Sciences 60.7 (2017): 714-720.

10. Li S., et al. "A pair of DICER1-positive monozygotic twins: One with pleuropulmonary blastoma, another with acute transient hepatitis". Case Reports Pediatrics Blood Cancer 64.12 (2017).

11. Fita AM., et al. "Type III pleuropulmonary blastoma in a dicer1 germline mutation carrier: The management of residual lung cystic lesions". Pediatric Blood Cancer 64.8 (2017).

12. Hill DA., et al. "DICER1 mutations in familial pleuropulmonary blastoma”. Science 325 (2009): 965.

13. Brenneman M., et al. "Temporal order of RNase IIIb and lossof-function mutations during development determines phenotype in DICER1 syndrome: a unique variant of the two-hit tumor suppression model". F1000Research 4 (2015): 214.

14. Pugh TJ., et al. "Exome sequencing of pleuropulmonary blastoma reveals frequent biallelic loss of TP53 and two hits in DICER1 resulting in retention of 5p-derived miRNA hairpin loop sequences". Oncogene 33 (2014): 5295-5302.

15. Murray MJ., et al. "Serum levels of mature microRNAs in DICER1-mutated pleuropulmonary blastoma". Oncogenesis 3 (2014): e87.

16. Addanki A., et al. "A case report of pleuropulmonary blastoma presenting as tension pneumothorax". Indian Journal of Medical and Paediatric Oncology 38 (2017): 70-72.

17. Sarangi PK., et al. "Bilateral pleuropulmonary blastoma (PPB) in a 2-year-old girl: A case report with review of literature". Journal of Medical and Allied Sciences 7.1 (2017): 67. 
18. Bobylev D., et al. "Giant pleuropulmonary blastoma”. European Journal of Cardiothoracic Surgery 50 (2016): 1215-1216.

19. Costa J., et al. "Fine needle aspiration in intraocular metastasis from pleuropulmonary blastoma. A case report and a review of the literature". Diagnostic Cytopathology 45.2 (2017): 156-160.

20. Priest JR., et al. "Cerebral metastasis and other central nervous system complications of pleuropulmonary blastoma". Pediatric Blood Cancer (2017).

21. Hashemi A., et al. "Pleuropulmonary blastoma in children: A case report". Iran Journal of Cancer Prevention 5 (2012): 105107.

22. Pinarli FG., et al. "Type II pleuropulmonary blastoma responsive to multimodal therapy". Pediatric Hematology and Oncology 22.1 (2005): 71-76.

23. Parsons SK., et al. "Aggressive multimodal treatment of pleuropulmonary blastoma". Annals of Thoracic Surgery 72.3 (2001): $939 \mathrm{e} 42$.

24. Romeo C., et al. "Pleuropulmonary blastoma: long-term survival and literature review". Medical and Pediatric Oncology 33.4 (1999): 372e6.

25. Wiliams GM., et al. "Effect of radiation on outcome of type II and type III PPB: A report from the international pleuropulmonary Blastoma Registry”. Journal of Clinical Oncology 30S (2012): 9521.

\section{Volume 4 Issue 7 July 2021}

(C) All rights are reserved by Abdelbasit Elsayed Ali., et al. 